\title{
Las visiones del alumnado sobre los Materiales Didácticos Digitales en España ${ }^{1}$
}

\section{As visões dos alunos sobre os Materiais Digitais Didáticos na Espanha}

\section{Student's visions about Digital Didactic Materials in Spain}

\author{
Laura Rego-Agraso* \\ Diana Marín Suelves**
}

\begin{abstract}
RESUMEN
El propósito fundamental de esta investigación ha sido conocer la percepción del alumnado de los últimos cursos de Educación Primaria sobre el uso de dispositivos tecnológicos y de los materiales didácticos digitales en las aulas. Para ello, se ha empleado metodología descriptiva y cualitativa, aplicando la entrevista grupal como instrumento de recogida de datos. Dichas entrevistas han sido realizadas a 11 alumnos de 2 comunidades autónomas del estado español - Galicia y Comunidad Valenciana. Los resultados muestran que el alumnado sigue mostrando preferencia por el libro impreso, aunque al mismo tiempo están hiperconectados y familiarizados con el uso personal y académico de los dispositivos electrónicos. Algunas de las potencialidades y dificultades del uso de los materiales didácticos digitales también fueron identificadas, así como una serie de propuestas de mejora desde la perspectiva de los principales usuarios/as de los materiales didácticos digitales.
\end{abstract}

Palabras clave: Tecnología. Recursos didácticos digitales. Percepción. Alumnado.

1 Los resultados de este estudio forman parte del proyecto "La escuela de la sociedad digital: análisis y propuestas para la producción y uso de los contenidos digitales educativos" [EDU2015-64593-R].

* Universidad da Coruña. Dpto. de Pedagoxía e Didáctica. Coruña, Galicia, España. E-mail: laura.rego@udc.gal.http://orcid.org/0000-0002-1660-4939.

** Universitad de València. Dpto. Didáctica y Organización Escolar. València, València, España. E-mail: diana.marin@uv.es. https://orcid.org/0000-0002-5346-8665. 


\title{
RESUMO
}

O objetivo fundamental desta pesquisa foi conhecer a percepção dos alunos dos últimos cursos da Educação Básica na Espanha sobre o uso de dispositivos tecnológicos e materiais didáticos digitais. Para isso, utilizou-se uma metodologia descritiva e qualitativa, aplicando a entrevista em grupo como instrumento de coleta de dados. Estas entrevistas foram conduzidas a 11 estudantes de duas comunidades autónomas do estado espanhol - Galícia e Comunidad Valenciana. Os resultados mostram que os alunos ainda preferem o livro impresso, mas ao mesmo tempo estão hiperconectados e familiarizados com o uso pessoal e acadêmico de dispositivos eletrônicos. Algumas das potencialidades e dificuldades do uso dos materiais didáticos digitais também foram identificadas, assim como uma série de propostas de melhoria na perspectiva dos principais usuários de materiais didáticos digitais.

Palavras-chave: Tecnologia. Recursos didáticos digitais. Percepção. Estudantes.

\begin{abstract}
The main objective of this research has been to know the perception of primary education students about the use of technological devices and digital teaching materials in their homes and classrooms. A descriptive and qualitative methodology has been used to achieve this objective, using a group interview to collect data. These interviews were conducted with 11 students from 2 autonomous regions of Spain - Galicia and Comunidad Valenciana. The results obtained show that students persist in choosing textbooks as their favourite didactic technology, but at the same time they are hyperconnected and habituated to the usual use of digital devices. Some strengths and difficulties in the use of digital devices were also found and some proposals for improvement are pointed out from the perspective of the main users of digital teaching materials.
\end{abstract}

Keywords: Technology. Digital didactic resources. Perception. Students.

\section{Introducción}

La sociedad actual se caracteriza por los continuos avances tecnológicos (Fuentes, López \& Pozo, 2019). El impacto de las tecnologías en todos los ámbitos de la vida cotidiana, sin excepción, en nuestro contexto, es una realidad, lo que ha supuesto una gran transformación de la sociedad en las últimas décadas (Reyero, 2019). En este mundo líquido, flexible y voluble (Bauman, 
2007) los cambios que se derivan de la introducción de las tecnologías afectan a la economía, el trabajo y las relaciones (Schwab \& Davis, 2018), a nuestro estilo de vida (Pedreño, 2015) y, por supuesto, no son ajenos a la educación. Es más, ésta es una de las áreas donde más potencialidad ha demostrado tener la tecnología (De Moya, Hernández, Hernández \& Cózar, 2011).

En los últimos años, muchos son los estudios que se han centrado en el papel de la tecnología en la escuela. Estas investigaciones han puesto el foco en el impacto de la incorporación de las TIC en la escuela, en diferentes contextos y etapas educativas, como la Educación Superior (Tapasco \& Giraldo, 2017), la Educación Secundaria (Fernández-Miravete, 2018) o la Educación Primaria (Martínez-Serrano, 2019; Toribio, 2019). Otros de los focos de atención han sido el grado de integración de las TIC (González, Espuny, De Cid \& Gisbert, 2012); la competencia digital docente (Fuentes, López \& Pozo, 2019) y la competencia digital del alumnado (Fernández-Miravete, 2018); la alfabetización digital (Cabero \& Llorente, 2008); las políticas educativas en este campo (Sanabria, Alvarez \& Peirats, 2017); el rol de gestores, docentes, administración o editoriales (ANELE, 2018; Peirats, Gallardo, San Martín \& Cortés, 2015); la brecha digital (Calderón, 2019); la percepción de los diferentes agentes en cuanto a la implantación y valoración de las tecnologías en la escuela (Martínez-Serrano, 2019; Sánchez, Alba \& Sánchez, 2018); el análisis de dispositivos (Carvalho, Luengo, Casas \& Cubero, 2018) como las tabletas digitales (Fernández-Rodrigo, 2016), o herramientas como los blogs educativos (Aznar \& Soto, 2010) o los webs educativos (Area, 2003). También se han realizado estudios sobre los materiales didácticos digitales (Vicente, Marín \& Cepeda, 2018; Mato, Castro \& Pereiro, 2018) y su evaluación (Cepeda, Gallardo \& Rodríguez, 2017), así como centrados específicamente en los libros digitales vs impresos (Carvalho, Luengo, Casas \& Cubero, 2018) o en los libros de texto digitales (Rodríguez-Rodríguez \& Rodríguez-Regueira, 2016). El desarrollo exponencial de materiales y recursos tecnológicos justifican la necesidad de análisis y reflexión sobre su introducción en la escuela. En este contexto, tras el estudio del estado del arte se concluye que los trabajos en los que se considera la visión del alumnado (Sánchez, Alba \& Sánchez, 2018) sobre los MDD son escasos.

Por otro lado, se ha producido una metamorfosis en los materiales educativos, "una mutación del relato cultural y de la funcionalidad pedagógica del material" (Area, 2017, p. 25) que va más allá de la digitalización de la información y de los contenidos curriculares. Esta investigación pretende explorar los fenómenos educativos que acompañan al proceso de cambio del modelo de uso de los materiales didácticos en el contexto escolar, por lo que interesa conocer las visiones de los agentes educativos sobre estos materiales y las prácticas de enseñanza y aprendizaje desarrolladas. 


\section{Metodología del estudio}

\section{Objetivos}

En el proyecto de investigación cuyos resultados se presentan aquí, se pretendió identificar las representaciones o visiones que los agentes educativos tienen sobre el potencial didáctico de los materiales didácticos digitales (de aquí en adelante, MDD) en la enseñanza primaria (Area, Hernández \& Sosa, 2016). El diseño es descriptivo-explicativo y se ha utilizado metodología cualitativa. En este artículo se presenta el estudio comparativo de las visiones del alumnado de 2 comunidades autónomas españolas: Galicia y Comunidad Valenciana.

\section{La muestra del estudio}

Los criterios tomados en consideración para seleccionar a los participantes fueron la diversidad de centros a los que asistían según su titularidad (concertados y públicos); su ubicación geográfica (centro ciudad, extrarradio o ayuntamientos rurales) y su grado de uso de las TIC en la escuela. Además, para la elección del alumnado se consideró que ambos sexos estuviesen representados, debiendo estar cursando todos ellos/as, $5^{\circ}$ o $6^{\circ}$ de Educación Primaria. La muestra estuvo compuesta por 11 alumnos; seis de Galicia y cinco de la Comunidad Valenciana; siete chicos y cuatro chicas; cuatro estaban en $5^{\circ}$ y siete en $6^{\circ}$; tres acudían a un centro concertado y ocho a un centro público. Las edades estaban comprendidas entre 10 y 12 años.

\section{Instrumentos de recogida de información}

Se utilizó como instrumento una entrevista grupal elaborada ad hoc para conocer la percepción del alumnado sobre el uso que hacen de los MDD, y sus características, tiempos y utilidades. El guion fue validado por un grupo de cuatro expertos - profesores universitarios especialistas en educación y TIC -. El instrumento está compuesto por 27 ítems divididos en cuatro bloques de contenido: datos de identificación (5), valoración de los MDD (4), uso personal (3) y uso académico (15).

\section{Análisis de los datos obtenidos}

Una vez transcritas las entrevistas y realizada una primera valoración de las mismas, para analizar los datos se han empleado dos matrices de contenido diseñadas ad hoc, con cuatro categorías generales de análisis correspondientes 
con los bloques del guion empleado en la entrevista grupal: 1. Descripción de la muestra; 2. Preferencias de uso entre lo digital y lo impreso; 3 . Uso personal de los dispositivos y MDD y 4. Uso académico. El análisis se realizó agrupando las aportaciones del alumnado en torno a estas categorías, codificando a partir de las transcripciones literales en la primera matriz y en la segunda realizando la interpretación en relación a los objetivos de investigación.

\section{Resultados}

\section{Valoración de los MDD}

En relación a las preferencias manifestadas por el alumnado a la hora de interactuar con materiales didácticos, una amplia mayoría, tanto de Galicia como de Valencia, señalan optar por el material en formato impreso:

[Es más fácil de seguir la lectura] si seguimos leyendo nosotros [no si alguien lee] (A_5G) [...] Entendemos mejor que si lo hace el ordenador [...] $\mathrm{Si}$ (Todos).

Entre las razones para realizar esta elección se alude, por un lado a que comprenden mejor los contenidos si los lee o explica un compañero/a o el propio profesor/a, que si lo hace un dispositivo electrónico, al tiempo que perciben que un uso en exclusiva de MDD conllevaría un incremento del trabajo en sus libretas. También indican que el material impreso es más fácil de conservar y no tienen tanto miedo a que se pueda romper como los dispositivos electrónicos. Como dificultad añadida al uso exclusivo del libro de texto digital, también se menciona la evaluación del trabajo del alumnado, dado que al no ser el profesorado el que corrige los ejercicios, esto puede dar lugar a algún inconveniente a la hora de poner las cualificaciones, dado que son conscientes de que existen otros elementos a considerar como su comportamiento en el aula.

En el alumnado de los centros en los que no se ha integrado las TIC en el aula se observa una actitud poco proclive a tener que descargar o buscar el material en la Tablet, ya que el libro de texto tradicional, para ellos, lo tiene todo. A pesar de ello, también existen alumnos que destacan que dispositivos como la Tablet, por ejemplo, permiten más búsquedas y conexiones que les facilitan el trabajo académico, además de que bajo la óptica de alguno de los/as participantes, estarían contribuyendo a respetar el medio ambiente al prescindir en mayor medida del papel. Todos ellos proceden de centros que emplean tecnología de forma habitual: 
Prefiero usar [la tableta] porque es mejor para el medio ambiente porque no se talan árboles, también porque hay aplicaciones que pueden ayudar a comprender mejor, también lo prefiero porque, si por ejemplo no te sabes una palabra, en [el buscador] puedes poner perfectamente el traductor y te lo traduce, o si no sabes el significado de una palabra en [la tableta] lo puedes tener y es mucho más cómodo (A_5V).

Por otro lado, con independencia de que en sus centros usen de forma cotidiana los MDD o no, el libro de texto impreso está omnipresente a lo largo de la entrevista en ambas comunidades. Las diferencias que establecen entre el libro digital y el libro de texto en papel son exclusivamente de carácter tecnológico, considerando que son lo mismo pero en distinto soporte: Tú coges el ordenador y [...] te registras y pones el código del libro y consigues el libro digitalmente que lo tienes en el ordenador (A_6G).

\section{Uso personal}

Todos los alumnos entrevistados cuentan en su hogar con recursos tecnológicos, lo que pone de manifiesto la extensión de los dispositivos asociados a las TIC en relación a la infancia. Tal como relatan, todos ellos cuentan en sus casas con televisión, ordenador (en su mayoría portátiles), Tablet/Ipad, videoconsola y teléfono móvil (la mayoría), con ratios superiores a 3 recursos por hogar en algunos casos y realizando algunos de ellos un uso compartido con la unidad familiar y otros, un uso en exclusividad:

Nosotros disponemos de muchos aparatos tecnológicos [...]. Ordenador, yo tengo uno, mis padres tienen otro. También tenemos 2 teles. Tenemos 3 móviles para los 3 [...] 4 tablets, solo que algunas están rotas [...]. También tenemos el móvil del trabajo de mi padre [...] sobre todo, solemos utilizar los móviles, la tele y el ordenador, para ellos trabajar y [...] yo para buscar información, ver vídeos en Youtube, etcétera (A_3V).

En general, conciben estos dispositivos como destinados a la ocupación de su tiempo libre y de ocio de una forma lúdica, aunque pueden servir también para realizar trabajos de aula. Estos dispositivos suelen tener conexión a Internet, y algunos y relatan que cuentan con perfiles en aplicaciones como Instagram, Facebook y WhatsApp en el caso de Galicia. También es interesante mencionar 
la presencia de la brecha digital en cuanto al acceso a Internet en las zonas rurales (Galicia). Esta realidad influye en la adquisición de dispositivos electrónicos por parte de las familias, dado que sin el acceso a la red, una gran parte de sus potencialidades se pierden: Yo tengo una tele. En mi casa no tenemos Internet porque en mi zona no hay mucha red y, yo y mi madre, compartimos el móvil (A_2V).

\section{Uso académico}

En cuanto al uso académico de los dispositivos electrónicos, existe una amplia diversidad entre los entrevistados/as. En el caso de Galicia, tres de los seis estudiantes afirman hacer uso directo de dispositivos digitales en sus aulas, bien a través de los libros de texto digital o con otras aplicaciones y dispositivos:

[...] Tienes que tener un usuario, ponerle un nombre, una contraseña, entras [...] y tu profesor [...] pone ejercicios y tú los tienes que ir haciendo y tienes de plazo lo que ponga alli [...]. [Quién corrige es] el ordenador, bueno la página (A_2G).

Los restantes aluden a que es el profesorado el que en ocasiones hace uso de los MDD en el aula, actuando ellos como observadores. En el caso de Valencia, la mayoría señalan usar, en general, ordenadores en el colegio, pero la Tableta/Ipad y el móvil se usan de forma esporádica. Como en el caso de Galicia, también se utiliza la PDI, aunque se destaca sobre todo su uso como proyector. En cuanto al poco uso de recursos como la Tableta, el alumnado alude a motivos relacionados con la ausencia de dichos recursos en el centro (hay pocas); las carencias técnicas que dichos recursos, en caso de existir, puedan presentar para ser usados (todavía no se han puesto a punto) y a la ausencia de conocimientos del profesorado en relación a su manejo. Lo que parece patente a lo largo de las entrevistas es que los materiales digitales conviven en las aulas con los materiales impresos tradicionales, fundamentalmente libros de texto, fichas y cuadernos, dejando entrever que se trabaja desde un modelo híbrido.

Por otro lado, en Galicia no se detectan diferencias en cuanto al uso de los MDD en el alumnado procedente de centros públicos y concertados, mencionando todos ellos recursos tecnológicos semejantes en sus aulas (ordenador, Tablet/ Ipad y PDI) y aplicaciones como Aula Planeta, Anaya Educación, Saber dixital o Minecraft. En el caso de Valencia sin embargo, si se detecta que la alumna del centro concertado, está mucho más familiarizada con el uso de dispositivos electrónicos y MDD que sus compañeros procedentes del centro público, dado 
que indica emplear el Ipad incluso para hacer exámenes y menciona muchas otras aplicaciones y utilidades del mismo (Savia digital, modo, blink, keynote, pages, Imovie o kahoot!).

En cuanto a la permisividad en el colegio de dispositivos electrónicos personales, casi la totalidad del alumnado entrevistado, tanto en Galicia como en Valencia señala que los dispositivos empleados en el colegio son propiedad del mismo y que no se les permite llevar sus propios dispositivos a la escuela:

No, no nos dejan [...] tenemos que estar concentrados en nuestro trabajo, en nuestros estudios, no tanto en el móvil. No nos dejan utilizarlo ni tampoco traerlo. Si lo traes, te lo confiscan y lo guardan hasta que viene alguien a [...] dirección a cogerlo (A_4V).

A diferencia de esta situación mayoritaria en ambos contextos geográficos, la alumna del colegio concertado dentro del grupo de Valencia, indica que la permisividad de su colegio en relación al uso de dispositivos propios es mayor. Señala que el móvil lo utiliza para asuntos personales en el contexto del colegio y no siempre académicos: Nos dejan llevar el móvil para [...] escuchar música, hacernos fotos y eso... Y el Ipad en el colegio lo podemos utilizar en clase y en la biblioteca con [...] (A_5V).

En relación a las preferencias de uso, el grupo de Galicia destaca que prefiere trabajar con el ordenador porque según su perspectiva, se adecúa mejor a las tareas que se les encomiendan en clase: se escribe mejor y más rápido y corrige ortográficamente. También destacan la tableta como recurso a emplear porque pesa menos y puede ser más manejable que muchos libros impresos de distintas materias: Si tienes que llevar lengua, lingua y mate [...] de deberes y te puedes llevar una tablet [...] pues yo prefiero la tecnología esa porque pesa menos (A_lG).

En relación a las aplicaciones, la preferencia está clara a la hora de elegir aquellas que describen como más divertidas y cuyo diseño gráfico es más atrayente. En este sentido, el alumnado de Valencia destaca aquellas que cuentan con efectos y movimientos, dibujos, música y juegos. Por otro lado, en trabajos académicos vinculados a matemáticas y música, este mismo grupo destaca blinklearning y Jclic.

Respecto a las ventajas una gran parte de los entrevistados alude a la motivación. El alumnado de Valencia destaca también otras particularidades como que comprenden mejor las explicaciones, pueden dejar notas, el trabajo es más rápido, pueden combinar texto con fotos o que el uso del corrector de textos 
les es útil para despreocuparse por las faltas de ortografía. También ven como una ventaja el hecho de que puedan estar realizando algún trabajo académico $y$, a la vez, realizar otras tareas.

En cuanto a las posibilidades de los MDD como promotoras de la comunicación entre compañeros/as y entre alumnado y profesorado, muchos de los entrevistados señalan que no favorecen la comunicación porque suelen emplearlas en muchas ocasiones para realizar trabajos de forma individual e incluso con auriculares. A pesar de ello, sí destacan que para realizar tareas o deberes fuera del contexto escolar contar con tecnología les facilita la comunicación y la realización de actividades al distribuirse las tareas entre ellos y poder simultanear su realización: Quedamos tres o cuatro en una biblioteca, llevamos los portátiles y uno está en word o en power point y otro busca información sobre una cosa y otro sobre otra. Acabamos antes (A_2G).

Sólo una alumna hace mención a que los MDD pueden facilitar la comunicación con el profesorado mediante el correo electrónico (A_5V) y pertenece a un centro concertado. Por último, con respecto a las dificultades en el uso de los MDD, las respuestas del alumnado varían considerablemente: desde aquellos que no destacan ningún elemento problemático en su uso hasta los que aluden a cuestiones como fallos técnicos de los dispositivos o de la batería, ausencia de conexión a la red o sobrecargas en la misma, responsabilidad en su cuidado, dificultades para seguir la lectura si la hace un dispositivo electrónico o que puedan ser una distracción en las tareas académicas que tienen encomendadas:

En clase si estás como asi [escondida] y la profesora no te ve puedes estar mirando fotos o jugando, cosa que es dificil porque ahora han encontrado una aplicación que es para vigilar lo que estás haciendo en el colegio (A_5V).

Destacan en todo caso, que los que mayor uso hacen, menos problemas señalan.

\section{Discusión}

La totalidad del alumnado participante en este estudio está en contacto con pantallas en diferentes momentos de su vida cotidiana. Están por lo tanto, 
moderadamente familiarizados con el uso de dispositivos electrónicos, tanto en sus hogares como en los centros educativos. Sin embargo, también es cierto que existen diferencias claras en la frecuencia de uso, en la disponibilidad de los propios recursos y en las distintas finalidades para las que se emplean.

En relación con las preferencias manifestadas por el alumnado en el uso de MDD, ellos/as mismos ponen de relieve su predilección mayoritaria por el material impreso, particularmente el libro de texto, lo que puede estar relacionado tanto con la necesidad de contacto humano a la hora de aprender o interiorizar contenidos, como con una mayor familiaridad con este recurso, cuyo uso sigue siendo muy extenso en las aulas españolas.

Esta predilección del alumnado de lo impreso frente a lo digital coincide con la reflejada en otros estudios llevados a cabo tanto en España (Carvalho, Luengo, Casas \& Cubero, 2018) como en otras latitudes (Jhangiani, Dastur, Le Grand \& Penner, 2018).

Actualmente el espacio de centralidad tradicionalmente ocupado por los libros de texto como recurso educativo cuasi-hegemónico, comienza a ser desplazado por otros materiales didácticos que cuentan con las TIC como soporte (Rego, Barreira \& Mariño, 2018). Sin embargo, su configuración y diseño parece organizado bajo parámetros didácticos similares al libro en formato papel (Cepeda, Gallardo \& Rodríguez, 2017), característica de la que parece ser plenamente consciente el alumnado entrevistado, especialmente al revelar que el libro de texto digital es, en esencia, lo mismo que el libro de texto impreso, pero en un soporte diferente.

En relación al uso personal de los dispositivos electrónicos, se constata que éste es sobre todo, de tipo lúdico y orientado a la ocupación del tiempo libre, lo que coincide parcialmente con lo establecido en otras investigaciones en relación al uso de las tecnologías digitales por infancia y adolescencia (González \& López, 2018). Por otro lado, la ratio de dispositivos electrónicos a disposición del alumnado es amplia en nuestro estudio, superando los 3 recursos por hogar para uso compartido o exclusivo, lo que coincide con lo expuesto por Torrecillas, Vázquez \& Monteagudo (2017, p. 100) cuando indican que "los hogares urbanos con menores escolarizados tienden a ser hogares hiperconectados con una fuerte presencia de dispositivos tecnológicos, tanto por su situación predominante en el escenario como por su diversidad y número". Igualmente las estadísticas nacionales al respecto (INE, 2018) muestran que en España los niños/as entre 10 y 15 años hacen un uso cotidiano de internet tanto desde su vivienda (94,9\%) como desde el centro educativo $(73,1 \%)$. En relación con esto, se detecta que el alumnado cuya vivienda no se localiza en entornos urbanos, muestra especiales dificultades de conexión, lo que pone sobre la mesa el efecto de la ya conocida 
brecha digital (Calderón, 2019) en el acceso a las funcionalidades TIC, especialmente en los entornos menos urbanizados (Morales, 2016).

En relación al uso académico, vemos que en la amplia mayoría de los centros educativos a los que asiste el alumnado entrevistado se hace un uso restringido de los dispositivos electrónicos, permitiendo únicamente el uso de aquellos que son propiedad del centro y no del alumnado. El ordenador y la PDI son los dos dispositivos que están presentes en el entorno académico en el caso de todos los entrevistados/as, siendo el uso de la tableta y el móvil, un poco más limitado, pero igualmente amplio. Esto coincide con los estudios que analizan el uso de dispositivos electrónicos por parte de menores de 16 años en distintos países (Mascheroni \& Cuman, 2014) y que concluyen que en Europa por ejemplo, entre el $30 \%$ y el $60 \%$ de los niños usan cada día tablets u ordenadores portátiles. De igual modo, este mismo estudio constata que aproximadamente un 13\% de los menores de 10 años utiliza Smartphone a diario. En el estudio que presentamos, no todos los alumnos/as cuentan con móvil en propiedad, pero si indican hacer uso de ellos al estar presentes en el contexto familiar. Las plataformas de contenidos asociadas al libro de texto digital y algunas aplicaciones también son usadas de forma habitual según el alumnado entrevistado. Destacan así, las plataformas de las grandes editoriales de libros de texto y otras aplicaciones como MineCraft.

Por otro lado, se constata también que los MDD comparten espacio en las aulas con los materiales impresos, particularmente el libro de texto, de tal forma que la mayor parte del alumnado entrevistado tiene interiorizado el uso de los MDD como un complemento o un facilitador del aprendizaje en algunas circunstancias, pero no como dispositivos que tengan que emplearse en exclusividad. En este sentido, las búsquedas de información, la realización de ejercicios on line o la posibilidad de realizar varias tareas al mismo tiempo - la multitarea o multitasking - , son mencionadas como las acciones que más realiza el alumnado con los MDD, entendiéndose también como ventajas de los mismos. De hecho, en el estudio de Sánchez-Antolín \& Blanco (2016), ya se establece que en las escuelas españolas tiende a seguir predominando el uso de materiales impresos, siendo las búsquedas de información o los ejercicios individuales on line, las actividades que más realiza el alumnado. De igual modo, que los MDD faciliten la multitarea desde el punto de vista del alumnado, se relaciona claramente con la existencia de este hábito también en el consumo mediático que realizan los jóvenes (Fernández, Masanet \& Figueras, 2016), caracterizado por la acumulación de acciones o hiperactividad mediática. A pesar de que los MDD faciliten esta multitarea y esto para ellos sea aparentemente positivo, también existen estudios (Courage et al., 2014, cit. en Raynaudo \& Borgobello, 2016, p. 55) que indican que "existe un coste cognitivo asociado 
con el cambio de tarea frecuente, que conlleva una degradación significativa en el desempeño". De igual modo, otros estudios (Levine et al. cit. en Raynaudo \& Borgobello, 2016, p. 55) constatan que "el uso de mensajería instantánea mientras se realiza una actividad académica se correlaciona con la sensación de distracción". Precisamente la conceptualización de los MDD como posibles distractores en el desarrollo de la actividad académica, es una de las desventajas también señaladas por el alumnado entrevistado. Igualmente los problemas de conexión, la durabilidad de las baterías o la responsabilidad en el cuidado de los dispositivos, son señaladas como posibles desventajas. Por último y coincidiendo con los resultados obtenidos en otros estudios (Conde, Ávila, Núñez \& Mirabent, 2015), la mayor parte de los entrevistados/as valoran la tecnología como no facilitadora de la comunicación entre compañeros al emplearse, sobre todo, para la realización de tareas individualizadas. Con todo, el alumnado que hace un uso más amplio de los MDD, tiende a realizar una valoración de los mismos más positiva.

\section{Conclusiones}

Con la realización de este estudio se pretendió analizar la visión que mostraba el alumnado de los últimos cursos de Educación Primaria en relación al uso personal y académico de los MDD. Los datos obtenidos indican en primer lugar, que una gran parte del alumnado sigue prefiriendo el formato impreso a la hora de elegir los materiales didácticos, al tiempo que el uso que indican hacer de los MDD es, en la mayoría de los casos, complementario y supeditado al uso del libro de texto en papel. Dicha complementariedad ya ha sido constatada en otros estudios semejantes (Sánchez-Antolín \& Blanco, 2016), lo que implica que los MDD conviven con los recursos impresos en la escuela, aunque la presión por la introducción de éstos últimos de forma masiva en la misma es cada vez más perceptible, tanto por el profesorado como por toda la comunidad educativa (Gutiérrez, Palacios \& Torrego, 2010). Dada la relevancia de lo manipulativo y lo experiencial en la etapa de Educación Primaria y la configuración de su currículo en España, la convivencia de ambos recursos y su uso complementario, no parece una opción desaconsejada, sino que puede ser positivo facilitar al alumnado una variedad de materiales que le permitan elegir qué formato le resulta más cómodo para la realización de sus tareas académicas (Carvalho, Luengo, Casas \& Cubero, 2018). 
En general, de las percepciones del alumnado sobre los MDD utilizados se desprende que en muchos casos lo único que ha cambiado es el soporte y que la organización de la información sigue siendo de tipo lineal (Area, 2003). El alumnado indica que emplea habitualmente el libro de texto digital - normalmente alojado en las plataformas de las grandes editoriales - pero lo asocian, tanto en su diseño como en las tareas que les propone, al libro de texto impreso. Por el contrario, el alumnado no habla de crear ellos mismos los materiales de aprendizaje o planificar las búsquedas, aunque estas aparezcan como una de las tareas esenciales que realizan con los MDD. De igual modo, del estudio se desprende que el uso mayoritario que hacen de las TIC es básicamente lúdico, no académico, lo cual puede ser debido a que, en general, se les hayan mostrado pocas posibilidades en este sentido y por lo tanto, las desconozcan. Por el contrario, en sus hogares las TIC parecen estar muy presentes, conviviendo la mayoría de ellos/as en entornos multipantalla e hiperconectados (Torrecillas, Vázquez \& Monteagudo, 2017) y siendo el ordenador portátil y la tablet, los dispositivos que más afirman emplear en su cotidianeidad (Mascheroni \& Cuman, 2014). En relación con esto, particular atención merece la progresiva familiarización del alumnado con la multitarea, algo que señalan como una de las ventajas del uso de los MDD. Sin embargo, existen estudios (Courage et al., 2014, cit. en Raynaudo \& Borgobello, 2016) que relacionan dicho hábito con la posibilidad de un desempeño peor y con una mayor sensación de distracción. Tampoco valoran la tecnología como facilitadora de la comunicación (Conde, Ávila, Núñez \& Mirabent, 2015), ya que, en general, la emplean de forma individual. Esto último pone en evidencia que posiblemente no se esté realizando un aprovechamiento de todas las potencialidades de los MDD: de lo que afirma el alumnado se desprende que en los centros se emplean metodologías y concepciones pedagógicas todavía asimiladas a la versión impresa de los recursos. El reto por lo tanto, sigue siendo la innovación en el modelo de enseñanza al introducir las TIC en el aula (Area, 2008) y el cambio metodológico (Vicente, Marín \& Cepeda, 2018). Por tanto, una de las claves es la formación crítica del profesorado en competencia digital (Fuentes, López \& Pozo, 2019), al tiempo que se mejora tanto en la dotación de recursos en los centros como en su actualización y mantenimiento, especialmente en los enclaves rurales.

En cuanto a las líneas de investigación futura quedan preguntas abiertas como, por ejemplo, si el uso habitual de los MDD mejora el rendimiento del alumnado o si el proceso educativo se ve enriquecido por las TIC, más allá del propio rendimiento. Las implicaciones de los MDD en el aprendizaje formal también deberían ser abordadas de forma específica, valorando especialmente su impacto cognitivo en la infancia, así como los riesgos conductuales asociados a las TIC y cómo convertirlos en potencialidades. 


\section{REFERENCIAS}

ANELE (2018). El libro educativo en España. Curso 2018-2019. Informe anual de ANELE (Asociación Nacional de Editores de Libros y Material de Enseñanza). Madrid: ANELE.

Area, M. (2003). De los webs educativos al material didáctico web. Revista Comunicación y Pedagogía, 188, 32-38.

Area, M. (2008). La innovación pedagógica con TIC y el desarrollo de las competencias informacionales y digitales. Revista de Investigación en la Escuela, 64, 5-17.

Area, M. (2017). La metamorfosis digital del material didáctico tras el paréntesis Gutenberg. RELATEC, 16(2), 13-28.

Area, M., Hernandez, V. \& Sosa, J. J. (2016). Models of educational integration of ICTs in the classroom. Comunicar, 47, 79-87.

Aznar, V. \& Soto, J. (2010). Análisis de las aportaciones de los blogs educativos al logro de la competencia digital. Revista de investigación en educación, 7, 83-90.

Bauman, Z. (2007). Los retos de la educación en la modernidad líquida. Madrid: Gedisa.

Cabero, J. \& Llorente, M. C. (2008). Del eLearning al Blended Learning: nuevas acciones educativas. Quaderns Digitals, 51, 1-8.

Calderón, D. (2019). Una aproximación a la evolución de la brecha digital entre la población joven en España (2006-2015). Revista Española de Sociología, 28(1), 27-44.

Carvalho, J. L., Luengo, R., Casas, L. \& Cubero, J. (2018). Para estudiar, ¿mejor el libro impreso o el libro digital?: un estudio exploratorio de naturaleza cualitativa. CIAIQ2018, 1.

Cepeda, O., Gallardo, I. M. \& Rodriguez, J. (2017). La evaluación de los materiales didácticos digitales. RELATEC, 16 (2), 79-95.

Conde, S., Ávila, J. A., Núñez, L. \& Mirabent, M. D. (2015). Opinión del profesorado y alumnado sobre la implantación, uso y resultados de las TIC en Educación Primaria: evaluación de un Centro. REICE, 13 (3), 57-75.

De Moya, M. D. V., Hernández, J. R., Hernández, J. A. \& Cózar, R. (2011). Análisis de los estilos de aprendizaje y las TIC en la formación personal del alumnado universitario a través del cuestionario REATIC. Revista de Investigación Educativa, 29 (1), 137-156.

Fernández-Rodrigo, L. (2016). El uso didáctico y metodológico de las tabletas digitales en aulas de educación primaria y secundaria de Cataluña. Pixel-Bit: Revista de medios y educación, 48, 9-25.

Fernández-Miravete, Á. (2018). La competencia digital del alumnado de Educación Secundaria en el marco de un proyecto educativo TIC (1: 1). EDUTEC. Revista Electrónica de Tecnología Educativa, 63, 60-72. 
Fernández, A., Masanet, M. J. \& Figueras, M. (2016). Tic i Joves. Reflexions i reptes per al treball educatiu (Col-lecció Aportacions, 54). Barcelona: Departament de Treball, Afers Socials i Famílies - Generalitat de Catalunya.

Fuentes, A., López, J. \& Pozo, S. (2019). Análisis de la Competencia Digital Docente: Factor Clave en el Desempeño de Pedagogías Activas con Realidad Aumentada. REICE. Revista Iberoamericana sobre Calidad, Eficacia y Cambio en Educación, 17 (2), 27-42.

González Martínez, J., Espuny Vidal, C., de Cid Ibeas, M. \& Gisbert Cervera, M. (2012). INCOTIC-ESO. Cómo autoevaluar y diagnosticar la competencia digital en la Escuela 2.0. Revista de investigación educativa, 30 (2), 287-302.

González-Ramírez, T. \& López-Gracia, A. (2018). La identidad digital de los adolescentes: usos y riesgos de las Tecnologías de la Información y la Comunicación. RELATEC, 17 (2).

Gutiérrez, A., Palacios, A. \& Torrego, L. (2010). La formación de los futuros maestros y la integración de las TIC en la educación: anatomía de un desencuentro. Revista de Educación, 352, 1-17.

Instituto Nacional de Estadística (INE) (2018). Niños de 10 a 15 años usuarios de ordenador, internet y lugar de uso de internet en los últimos 3 meses. Recuperado de: https://www.ine.es/jaxi/Tabla.htm?path=/t25/p450/base_2011/a2018/10/\&file=04067. px\&L=0. Acceso en: mar. 2019.

Jhangiani, R. D., Dastur, F. N., Le Grand, R. \& Penner, K. (2018). As Good or Better than Commercial Textbooks: Students' Perceptions and Outcomes from Using Open Digital and Open Print Textbooks. The Canadian Journal for the Scholarship of Teaching and Learning, 9 (1).

Martínez-Serrano, M. C. (2019). Percepción de la Integración y uso de las Tecnologías de la Información y la Comunicación (TIC). Estudio de Profesores y Estudiantes de Educación Primaria. Información tecnológica, 30 (1), 237-246.

Mascheroni, G. \& Cuman, A. (2014). Net Children Go Mobile: Final Report. Milano: Educatt.

Mato-Vázquez, D., Castro, M. M. \& Pereiro, M. C. (2018). Análisis de materiales didácticos digitales para guiar y/o apoyar el proceso de enseñanza-aprendizaje de las matemáticas. @tic: Revista D’Innovació Educativa, 20, 72-79.

Morales, N. (2016). Las TIC y los escolares del medio rural: entre la brecha digital y la educación inclusiva. Bordón, 69 (3), 41-56. DOI: 10.13042/Bordon.2017.52401

Pedreño, A. (2015). Disrupción digital. Un inmenso potencial para las universidades. Telos, 101.

Peirats, J., Gallardo, I. M., San Martín, Á. \& Cortés, S. (2015). Los contenidos curriculares digitalizados: Voces y silencios en el ámbito editorial. Educatio siglo XXI, 33 (3), 39-62. 
Raynaudo, G. \& Borgobello, A. (2016). Uso de tic: posibles relaciones con habilidades cognitivas e interpersonales en un grupo de adolescentes. Ciencia, Docencia y Tecnología, 27 (53), 50-74.

Rego, L., Barreira, E. M. \& Mariño, R. (2018). La representación de la diversidad social en los materiales didácticos digitales. @tic: Revista D'Innovació Educativa, 20, 63-71. http://doi.org/10.7203/attic.20.12118

Reyero, M. (2019). La educación constructivista en la era digital. Revista Tecnología, Ciencia y Educación, 12, 111-127.

Rodríguez-Rodríguez, J. \& Rodríguez-Regueira, N. (2016). Revisión de la investigación publicada sobre el libro de texto digital en revistas, publicaciones y congresos internacionales de referencia. Profesorado. Revista de Currículum y Formación de Profesorado, 20 (1), 9-31.

Sanabria, A. L., Alvarez, Q. \& Peirats, J. (2017). Las políticas educativas en la producción y distribución de materiales didácticos digitales. RELATEC, 16 (2), 63-77.

Sánchez, J. M., Alba, C. \& Sánchez, P. (2018). Valoraciones del alumnado de Educación Primaria sobre lecturas digitales diseñadas con UDL Book-Builder como apoyo en los procesos lectores. Aula abierta, 47(4), 481-490.

Sánchez-Antolín, P. \& Blanco, M. (2016). La política educativa TIC de la Comunidad de Madrid (España): la perspectiva del profesorado. RELATEC: Revista Latinoamericana de Tecnología Educativa, 15(1), 45-58.

Schwab, K. \& Davis, N. (2018). Shaping the Future of the Fourth Industrial Revolution. New York: Currency.

Tapasco, O. A. \& Giraldo, J. A. (2017). Estudio Comparativo sobre Percepción y uso de las TIC entre Profesores de Universidades Públicas y Privadas. Formación Universitaria, 10(2), 3-12.

Toribio, M. C. (2019). Importancia del uso de las TIC en educación primaria. Atlante Cuadernos de Educación y Desarrollo, febrero. Recuperado de: https://www.eumed.net/ rev/atlante/2019/02/uso-tic-primaria.html. Acceso en: mar. 2019.

Torrecillas, T., Vázquez, T. \& Monteagudo, L. (2017). Percepción de los padres sobre el empoderamiento digital de las familias en hogares hiperconectados. El Profesional de la Información, 26(1), 97-104.

Vicente, R. M., Marín, D. \& Cepeda, O. (2018). Análisis de materiales didácticos musicales para Primaria en la escuela digital. Revista Electrónica LEEME, 42.

Texto recibido el $05 / 08 / 2019$.

Texto aprobado el 02/09/2019. 